\title{
SPHERICAL HARMONICS AND INTEGRAL GEOMETRY ON PROJECTIVE SPACES
}

\author{
BY
}

ERIC L. GRINBERG

\begin{abstract}
The Radon transform $R$ on $\mathbf{C} P^{\mathrm{n}}$ associates to a point function $f(x)$ the hyperplane function $R f(H)$ by integration over the hyperplane $H$. If $R^{t}$ is the dual transform, we can invert $R^{t} R$ by a polynomial in the Laplace-Beltrami operator, and verify the formula of Helgason [7] with very simple computations.

We view the Radon transform as a $G$-invariant map between representations of the group of isometries $G=U(n+1)$ on function spaces attached to $\mathbf{C} P^{n}$. Pulling back to a sphere via a suitable Hopf fibration and using the theory of spherical harmonics, we can decompose these representations into irreducibles. The scalar by which $R$ acts on each irreducible is given by a simple integral. Thus we obtain an explicit formula for $R$. The action of $R^{t} R$ is immediately related to the spectrum of C $P^{n}$. This shows that $R^{t} R$ can be inverted by a polynomial in the Laplace-Beltrami operator. Similar procedures give corresponding results for the other compact 2-point homogeneous spaces: $\mathbf{R} P^{n}, \mathbf{H} P^{n}, \mathbf{O} P^{n}$, as well as spheres.
\end{abstract}

0. Introduction. In 1917 Radon showed that a function in Euclidean space could be recovered from its integrals over hyperplanes and thereby defined the now celebrated Radon transform. It associates to a point function $f(x)$ the hyperplane function $R f(H)=\int_{H} f$. This type of transform arises naturally in many geometric situations - whenever a nice family of submanifolds (such as hyperplanes) is present. Thus Helgason considered the Radon transform in the setting of homogeneous spaces [8], and Gelfand in a quite general topological setting [3]. In particular, Helgason studied the cases of compact two-point homogeneous spaces using the theory of antipodal manifolds. Since (except for spheres) these spaces are projective spaces, they can also be studied using projective geometry and algebra.

In this paper we consider the Radon tranform on compact two-point homogeneous spaces from the point of view of group representations. The Peter-Weyl theory for compact Lie groups makes the analysis very simple. Thus we can derive important properties of the Radon transform with only a few computations, and these computations bring out the nature of the geometry involved and the behaviour of the Radon transform under it. In particular, we can recover the inversion formulae of Helgason involving the Laplace-Beltrami operator [7]. This was already done by Guillemin for the sphere $S^{2}$ [5]. Therefore, we skip the cases of spheres and also real projective spaces (which are essentially the same). In fact, the analysis for

Received by the editors February 8, 1982 and, in revised form, September 3, 1982. Presented to the Society, January 14, 1982 at the Cincinnati meeting.

1980 Mathematics Subject Classification. Primary 43A85, 43A90, 58G15.

Key words and phrases. Integral geometry, Radon transform, spherical harmonics, projective spaces. 
complex projective space makes it clear what needs to be done for spheres. On the other hand, the complex and quaternionic cases can be handled by reducing to spheres using the Hopf fibration, and this we show in detail.

There is also the exceptional case of the Cayley projective plane. Although there is no appropriate Hopf fibration here, everything needed for the representation theory is present, and analogous results can be derived. The same technique ought to work for other homogeneous spaces such as Grassmannians, and it may be useful in answering questions of admissibility (see Gelfand et al., Generalized functions [4], for a discussion of the problem of admissibility). Perhaps some of these ideas will be included in a later paper. Added in proof: see also [14].

We are indebted to Victor Guillemin and Todd Quinto for their encouragement and helpful comments.

1. Decomposition of $L^{2}$ functions on $\mathbf{C} P^{n}$. We make us of the Hopf fibration $\pi$ : $S^{2 n+1} \rightarrow \mathbf{C} P^{n}$ and the standard theory of spherical harmonics to describe functions on $\mathbf{C} P^{n}$. Throughout the discussion we assume $n>1$, because the 2-point homogeneous space $\mathbf{C} P^{1}$ is best viewed as the sphere $S^{2}$. Also, we will often view $\mathbf{C} P^{n}$ as the homogneous space $U(n+1) / U(n) \times U(1)$, or equivalently as $S^{2 n+1} / U(1)$.

A function $f \in C\left(\mathbf{C} P^{n}\right)$ lifts to a function $\pi^{*} f \in C\left(S^{2 n+1}\right)$ which is homogeneous of degree zero with respect to the action of the circle group $S^{1}$ on $S^{2 n+1}$ (induced from the action on $\mathbf{C}^{n+1}$ ). The classical theory of spherical harmonics gives the following decomposition for $L^{2}\left(S^{2 n+1}\right)$ :

$$
L^{2}\left(S^{2 n+1}\right)=\bigoplus_{m=0}^{\infty} H_{m}(2 n+2)
$$

where $H_{m}(k)$ is the finite-dimensional vector space of harmonic polynomials homogeneous of degree $m$ in $k$ real variables restricted to $S^{k-1}$ (everything is complexvalued here). See Dieudonné [2, \$7] for a discussion of this decomposition. Our argument below will emulate this discussion.

Using the complex structure on $\mathbf{R}^{2 n+2}$ we can expand any polynomial in $x_{1}, \ldots, x_{2 n+2}$ as a polynomial in $z_{1}, \bar{z}_{1}, \ldots, z_{n+1}, \bar{z}_{n+1}$. Thus we get a 'Hodge decomposition' for the space of polynomials in $2 n+2$ real variables homogeneous of degree $m$ :

$$
P_{m}(2 n+2)=\bigoplus_{k=0}^{m} P_{(k, m-k)}(n+1)
$$

where $P_{(k, l)}(n+1)$ is the space of polynomials in $z_{0}, \ldots, z_{n}, \bar{z}_{0}, \ldots, \bar{z}_{n}$ homogeneous of degree $k$ in the $z$ 's and $l$ in the $\bar{z}$ 's. The real Laplacian

$$
\Delta_{\mathbf{R}}=\sum_{i=0}^{2 n} \frac{\partial^{2}}{\partial x_{i}^{2}}
$$

on $P_{m}(2 n+1)$ equals the complex Laplacian

$$
\Delta_{\mathbf{C}}=4 \sum_{i=0}^{m} \frac{\partial^{2}}{\partial z_{i} \partial \bar{z}_{i}}
$$


there, and $\Delta_{\mathbf{C}}$ 'commutes' with the Hodge decomposition for $P_{m}$ (in an obvious sense) so we get a Hodge decomposition for the space $H_{m}(2 n+2)$ as well:

$$
H_{m}(2 n+2)=\bigoplus_{k=0}^{m} H_{(k, m-k)}(n+1) .
$$

A polynomial $P \in P_{(k, l)}(n+1)$ is homogeneous of degree zero w.r.t. the dilation group $S^{1}=\left\{e^{i \theta}\right\}$ if and only if $k=l$. Such a polynomial $P$ can be viewed as a continuous function on $\mathbf{C} P^{n}$ : if $\left[z_{0}, \ldots, z_{n}\right]$ are homogeneous coordinates for a point in $\mathbf{C} p^{n}$ with $z_{0} \bar{z}_{0}+\cdots+z_{n} \bar{z}_{n}=1$, the value $P\left(z_{0}, \ldots, z_{n}\right)$ does not depend on the choice of coordinates. Thus one would suspect that the following orthogonal decomposition holds:

$$
L^{2}\left(\mathbf{C} P^{n}\right)=\bigoplus_{m=0}^{\infty} H_{(m, m)}(n+1) .
$$

The harmonic analysis leading up to this decomposition is well known (see Smith [11]). However, we will give an independent proof.

The summands in the right-hand side of (1) form a selfadjoint vector space of functions. In fact, they form an algebra. To see this, we recall the following decomposition of polynomials in $\mathbf{R}^{2 n+2}$ (Dieduonné [2, §7]):

$$
P_{m}(2 n+2)=H_{m}(2 n+2) \oplus r^{2} \cdot H_{m-2}(2 n+2) .
$$

(Here $r^{2}=x_{0}^{2}+\cdots+x_{2 n-1}^{2}=z_{0} \bar{z}_{0}+\cdots+z_{n} \bar{z}_{n}$.) This of course specializes to give the complex decomposition

$$
P_{(m, m)}(n+1)=H_{(m, m)}(n+1) \oplus r^{2} \cdot H_{(m-1, m-1)}(n+1) .
$$

Thus any function of $\mathrm{C} P^{n}$ which is represented by a polynomial $P$ in $z_{0}, \ldots, z_{n}$, $\bar{z}_{0}, \ldots, \bar{z}_{n}$ (homogeneous of degree zero w.r.t. $S^{1}$ ) is a finite sum of functions in the spaces $\left\{H_{m, m}\right\}$. Thus the right-hand side of (1) (as an inductive sum) forms a selfadjoint algebra of continuous functions on $\mathbf{C} P^{n}, \mathbb{Q}$. The action $U(n+1)$ on $\mathrm{C}^{n+1}$ induces an action on this algebra, preserving the decomposition (1). To show that $Q$ separates points on $C P^{n}$ is simple: by transitivity of the action of $U(n+1)$ on $\mathbf{C} P^{n}$, we can assume that one point is $[0, \ldots, 0,1]$ in homogeneous coordinates. Then

$$
P(z)=\sum_{i=0}^{n-1} z_{i} \bar{z}_{i} \in P_{(1,1)}(n+1)
$$

is zero at $[0, \ldots, 0,1]$ and nowhere else on $\mathbf{C} P^{n}$. Since $\mathcal{Q}$ contains constants, it vanishes at no point in $\mathbf{C} P^{n}$. By the Stone-Weierstrass theorem, the uniform closure of $\Theta$ contains all continuous functions on $\mathbf{C} P^{n}$, hence the $L^{2}$ closure contains all $L^{2}$ functions. This, together with the standard decomposition of $L^{2}\left(S^{2 n+1}\right)$, verifies (1).

We already know that the various subspaces in the R.H.S. of (1) are invariant under $U(n+1)$. Let us show them to be irreducible. Again, we emulate the case of ordinary spherical harmonics and use the idea of spherical functions as in Dieudonné [2].

$U(n+1)$ acts transitively on $S^{2 n+1}$. The isotropy group of a point (which we think of as the north pole) is isomorphic to $U(n)$, so $S^{2 n+1}=U(n+1) / U(n)$. 


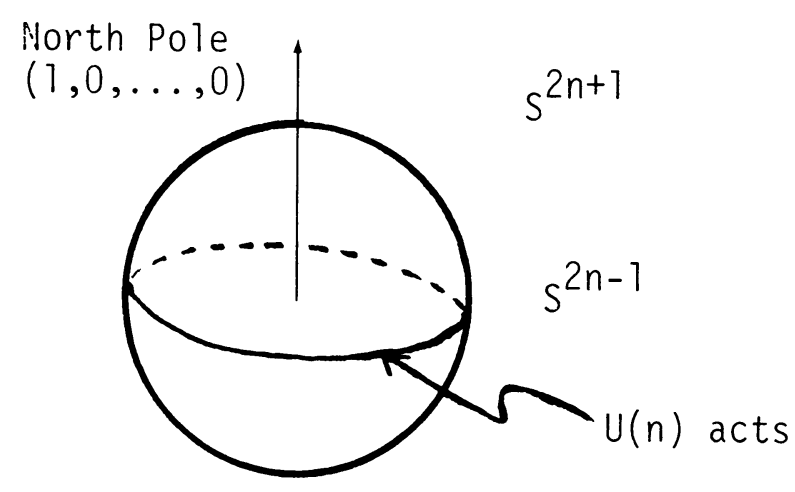

FIGURE 1

We show that each subspace $H_{(m, m)}(n+1)$ contains a unique spherical function, i.e., a function $f(x) \in \mathbf{C}\left(S^{2 n+1}\right)$ with value 1 at the north pole and invariant under the action of $U(n)$. Let $P(z) \in P_{(m, m)}(n+1)$. Write

$$
P(z)=\sum_{k, l=0}^{m} z_{0}^{k} \bar{z}_{0}^{l} g_{k, l}(w)
$$

where $w$ represents the remaining variables $z_{1}, \ldots, z_{n}, \bar{z}_{1}, \ldots, \bar{z}_{n}$, and $g_{k, l}$ is a polynomial in $w$ homogeneous of degree $m-k$ in $z_{1}, \ldots, z_{n}$ and degree $m-l$ in $\bar{z}_{1}, \ldots, \bar{z}_{n}$. Let $\Delta$ be the renormalized Laplacian

$$
\Delta=\frac{1}{4} \Delta_{\mathbf{C}}=\sum_{j=0}^{n} \frac{\partial^{2}}{\partial z_{j} \partial \bar{z}_{j}} .
$$

Then,

$$
\Delta P(z)=\sum_{k, l=1}^{m} k \cdot l \cdot z_{0}^{k-1} \bar{z}_{0}^{l-1} g_{k, l}(w)+\sum_{k, l=0}^{m} z_{0}^{k} \bar{z}_{0}^{l} \Delta g_{k, l}(w) .
$$

Now assume $P$ is harmonic. Looking at homogeneous parts gives

$$
(k+1)(l+1) g_{k+1, l+1}(w)+\Delta g_{k, l}(w)=0 \quad(0 \leqslant k, l \leqslant m-1) .
$$

Thus one can choose $g_{(0,0)}, g_{(0,1)}, \ldots, g_{(0, m)}, g_{(1,0)}, \ldots, g_{(m, 0)}$ arbitrarily and then the rest of the $g$ are decided by (3). We sketch this schematically below in Figure 2. Assume that $P$ is, in addition, a spherical function. Then in (2) each $g_{k, l}$ must be invariant under $U(n)$ (since $U(n)$ preserves the $z_{0}$ and $\bar{z}_{0}$ coordinates), so we must have $g_{k, l}=0$ (if $k \neq l$ ).

This is because $U(n)$ acts transitively on $S^{2 n-1}$, the sphere in $w$ space, and any invariant polynomial is a polynomial in the $w$-radius, $z_{1} \bar{z}_{1}+\cdots+z_{n} \bar{z}_{n}=|w|^{2}$. By (3), only $g_{(0,0)}$ may be specified. But $g_{(0,0)}$ is of type $(m, m)$ so $g_{(0,0)}(w)=c|w|^{2 m}$ where $c$ is decided by the condition $P$ (north pole) $=1$ and (2). 


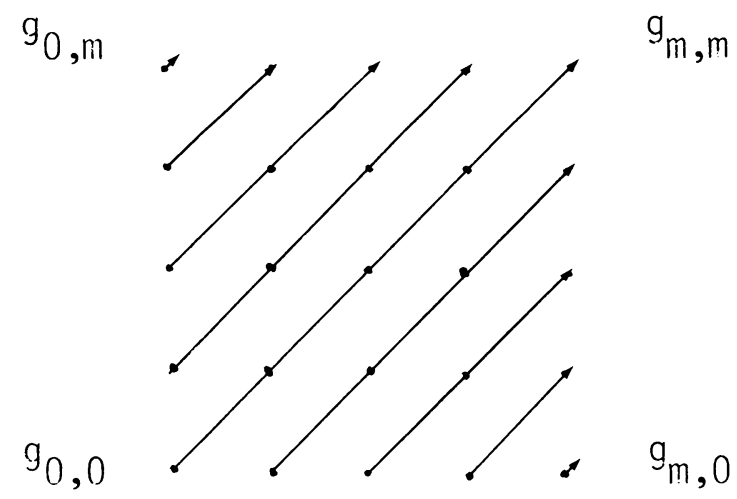

Figure 2

The existence of spherical functions in each $U(n+1)$-invariant subspace (of finite dimension, say) follows from the usual argument: take a function $f(x) \neq 0$. By transitivity of the action of $U(n+1)$ on $S^{2 n+1}$, we may assume $f$ is 1 at the north pole. Now averaging w.r.t. $U(n)$ we obtain a spherical function in our subspace. Since $H_{(m, m)}$ is $U(n+1)$-invariant and contains a unique spherical function, it is irreducible. The dimension of $H_{(m, m)}$ can be computed from the discussion following (3). We do not need this explicitly. Let us note, however, that $\operatorname{dim} H_{(k, k)}$ increases with $k$ (this is clear from (3) and Figure 2), so the $U(n+1)$ representations $\left\{H_{(k, k)}\right\}$ are pairwise inequivalent.

2. Invariant measures on $\mathbf{C} P^{n}$. The space $\mathbf{C} P^{n}$ is a homogeneous space of the group $G=U(n+1)$ and as such can be given a $G$-invariant metric, unique up to a normalization (we used the existence of such a metric implicitly in $\S 1$ ). One way to describe this metric is via the Hopf fibration $\pi$ :

$$
\begin{array}{r}
S^{1} \rightarrow \quad S^{2 n+1} \\
\downarrow \pi \\
\\
\\
\mathrm{C} P^{n}
\end{array}
$$

If we think of the sphere $S^{2 n+1}$ as imbedded in $\mathbf{C}^{n+1} \approx \mathbf{R}^{2 n+2}$, it has a natural inherited metric. The circle group $S^{1}=\left\{e^{i \theta} \mid \theta \in \mathbf{R}\right\}$ acts on $S^{2 n+1}$, as a group of isometries, multiplying each complex coordinate by the phase factor $e^{i \theta}$. The quotient space is $\mathbf{C} P^{n}$ and the Hopf fibration $\pi$ is the quotient map $S^{2 n+1} \rightarrow$ $S^{2 n+1} / S^{1}$. To define a metric on $\mathbf{C} P^{n}$, let $x \in \mathbf{C} P^{n}, v \in T_{x}\left(\mathbf{C} P^{n}\right)$. It is enough to define the length $g_{x}(v, v)$. Choose lifts $\tilde{x} \in S^{2 n+1}$ and $\tilde{v} \in T_{\tilde{x}}\left(S^{2 n+1}\right)$ so $x=\pi \tilde{x}$, $v=\pi_{*} \tilde{v}$. Let

$$
g_{x}(v, v)=\tilde{g}_{\tilde{x}}(\tilde{v}, v)
$$

where $\tilde{g}$ on the R.H.S. (4) denotes the metric on $S^{2 n+1}$. Distinct choices of lifts differ by a motion of $S^{1}$ which is an isometry. Thus the R.H.S. (4) does not depend on the choice of lifts and the metric $g$ is well defined. It is smooth because locally we can find smooth sections of $\pi$, so that the R.H.S. (4) depends smoothly on $(x, v)$. It is 
$G$-invariant because the metric on $S^{2 n+1}$ is $O(2 n+2)$-invariant and the $U(n+1)$ action imbeds in the $O(2 n+2)$ action.

It will be useful to have a description of the volume form associated with this metric in local coordinates. Let $\left[z_{0}, \ldots, z_{n}\right]$ denote homogeneous coordinates for a point in $\mathbf{C} P^{n}$. We have the standard affine open set

$$
U_{0}=\left\{\left[z_{0}, \ldots, z_{n}\right] \in \mathbf{C} P^{n} \mid z_{0} \neq 0\right\} .
$$

On $U_{0}$ we have affine coordinates $t_{i}=z_{i} / z_{0}(i=1, \ldots, n)$. In these coordinates the metric can be expressed as

$$
d s^{2}=c_{n} \frac{\left(1+|t|^{2}\right)\left(d t_{1} \overline{d t}_{1}+\cdots+d t_{n} \overline{d t}_{n}\right)-\left(\sum_{i} t_{i} d t_{i}\right)\left(\sum_{j} \bar{t}_{j} d \bar{t}_{j}\right)}{\left(1+|t|^{2}\right)^{2}}
$$

(where $c_{n}$ is a constant depending on $n$ and $|t|=t_{1} \bar{t}_{1}+\cdots+t_{n} \bar{t}_{n}$ ). See, for example, Kobayashi and Nomizu [9, vol. 2, p. 116].

This is, of course, the Fubini-Study metric on $\mathbf{C} P^{n}$. However, all that interests us here is the associated volume form

$$
d v=c_{n}^{\prime} \frac{d t_{1} \wedge \cdots \wedge d t_{n} \wedge d \bar{t}_{1} \wedge \cdots \wedge d \bar{t}_{n}}{\left(1+|t|^{2}\right)^{n+1}}=c_{n}^{\prime} \frac{d t \wedge \overline{d t}}{\left(1+|t|^{2}\right)^{n+1}} .
$$

The volume form (6) can be deduced from (5) by computing a determinant, or, one can verify directly that (6) is $G$-invariant. To see the latter, consider first transformations $g \in U(n+1)$ which leave the $z_{0}$ coordinate fixed, say

$$
g=\left(\begin{array}{l|l}
1 & 0 \\
\hline 0 & U
\end{array}\right) \quad \text { where } U \in U(n) .
$$

Then $g$ acts as the unitary matrix $U$ on the affine coordinates $t$. For a coordinate measure $d V=F(t) d t \wedge d \bar{t}$ to be invariant under all such $g, F$ must depend on $|t|$ only, for we have $g^{*} d V=F(U t) d t \wedge d \bar{t}$ and this must equal $d V$ for all $U$. Now $U(n+1)$ is generated by elements of the form $g$ above, and by motions in the $z_{0}, z_{1}$ plane, say

$$
g=\left(\begin{array}{cc|c}
\alpha & \beta & \\
-\bar{\beta} & \bar{\alpha} & \\
\hline & & I
\end{array}\right) \in U(n+1) \quad\left(\alpha, \beta \in \mathbf{C} ;|\alpha|^{2}+|\beta|^{2}=1\right) .
$$

To check that $\left(1+|t|^{2}\right)^{-(n+1)} d t \wedge d \bar{t}$ is $U(n+1)$-invariant it now suffices to show that it is preserved by motions of the type $g$ above. Now $g$ transforms the affine coordinates $t=\left(t_{1}, \ldots, t_{n}\right)$ to the affine coordinates $s=\left(s_{1}, \ldots, s_{n}\right)$ as follows:

$$
s_{1}=\frac{-\bar{\beta} z_{0}+\bar{\alpha} z_{1}}{\alpha z_{0}+\beta z_{1}}=\frac{-\bar{\beta} z_{0}+\bar{\alpha} t_{1} z_{0}}{\alpha z_{0}+\beta t_{1} z_{0}}=\frac{-\bar{\beta}+\bar{\alpha} t_{1}}{\alpha+\beta t_{1}},
$$

and similarly, $s_{i}=t_{i} /\left(\alpha+\beta t_{1}\right)(i=2, \ldots, n)$. Also,

$$
d s_{1}=\frac{d t_{1}}{\left(\alpha+\beta t_{1}\right)^{2}}, \quad d s_{i}=\frac{d t_{i}}{\alpha+\beta t_{1}}+a_{i} d t_{1} \quad(i=2, \ldots, n)
$$


and

$$
d s \wedge d \bar{s}=\frac{d t \wedge d \bar{t}}{\left|\alpha+\beta t_{1}\right|^{2 n+2}}
$$

Finally,

$$
\begin{aligned}
& \frac{d s \wedge}{\left(1+|s|^{2}\right)^{n+1}} \\
& \quad=\frac{d t \wedge d \bar{t}}{\left(1+\left|\left(-\bar{\beta}+\bar{\alpha} t_{1}\right) /\left(\alpha+\beta t_{1}\right)\right|^{2}+\sum_{i=2}^{n}\left|t_{i} /\left(\alpha+\beta t_{i}\right)\right|^{2}\right)\left(\alpha+\beta t_{1}\right)^{2 n+2}} \\
& =\frac{1}{\left(\left(|\alpha|^{2}+|\beta|^{2}+\left|t_{1}\right|^{2}+\cdots+\left|t_{n}\right|^{2}\right) /\left|\alpha+\beta t_{1}\right|^{2}\right)^{n+1}} \frac{d t \wedge d \bar{t}}{\left|\alpha+\beta t_{1}\right|^{2 n+2}} \\
& =\frac{d t \wedge d \bar{t}}{\left(1+|t|^{2}\right)^{n+1}} \cdot \text { Q.E.D. }
\end{aligned}
$$

3. The Radon transform on $\mathbf{C} P^{n}$. The Grassmannian of hyperplanes in $\mathbf{C} P^{n}$, $\operatorname{Gr}(n-1, n)$ can be viewed as the dual space $\mathbf{C} P^{n *}$. Under this duality, the point $\left[z_{0}, \ldots, z_{n}\right] \in \mathbf{C} P^{n}$ is identified with the hyperplane

$$
\left\{\left[w_{0}, \ldots, w_{n}\right] \in \mathbf{C} P^{n} \mid z_{0} w_{0}+\cdots+z_{n} w_{n}=0\right\} .
$$

Let $f(x)$ be a continuous function of points on $\mathbf{C} P^{n}$. We define its Radon transform $R f$ as a continuous function of hyperplanes in $\mathbf{C} P^{n}$ :

$$
R f(H)=\int_{h} f(x) d \mu_{H}(x) \quad\left(h \in \mathbf{C} P^{n *}\right)
$$

where $d \mu_{H}$ is the invariant measure on the hyperplane $H$ induced from the Fubini-Study metric on $\mathbf{C} P^{n}$. See Helgason [8] for general information about $R$.

Now $U(n+1)$ acts on $\mathbf{C} P^{n}$ and $\mathbf{C} P^{n *}$ by left translation, so $C\left(\mathbf{C} P^{n}\right)$ and $C\left(\mathbf{C} P^{n *}\right)$ are two representation spaces for $G=U(n+1)$. The Radon transform is a $G$-invariant map between these:

$$
\begin{aligned}
g(R f)(H) & =R f\left(g^{-1} H\right)=\int_{g^{-1} H} f(x) d \mu_{g^{-1} H}(x) \\
& \left.=\int_{H} f\left(g^{-1} x\right) d \mu_{H}(x) \quad \text { (by } G \text {-invariance of the measures } d \mu_{H}\right) \\
& =R(g f)(H) .
\end{aligned}
$$

We identify $\mathbf{C} P^{n}$ with $\mathbf{C} P^{n *}$ henceforth. By Schur's lemma, $R$ acts as a scalar multiple of the identity on each irreducible subrepresentation of $C\left(\mathbf{C} P^{n}\right)$. From $\S 1$ we know that the irreducible subspaces in question are just the $H_{(k, k)}(k=0,1, \ldots)$. To invert the Radon transform on $\mathbf{C} \boldsymbol{P}^{n}$, it is enough to compute the scalar $c(k)$ which represents $R$ on $H_{(k, k)}$. To find $c(k)$ we select a function $f \in H_{(k, k)} \backslash 0$ and 
evaluate the ratio $R f / f$. Take

$$
f([z])=f\left(\left[z_{0}, \ldots, z_{n}\right]\right)=\left(z_{0} \bar{z}_{1}\right)^{k} \quad\left(|z|^{2}=1\right) .
$$

This $f$ happens to be a highest weight vector for the representation $H_{k, k}$. We chose it because $R f$ is particularly simple to evaluate. Any other $f \neq 0$ would work, but the integral might be more complicated.

Though tempting, we must be careful not to evaluate $f$ on $[1,1,0, \ldots, 0]$ and obtain 1 , because $|(1,1,0, \ldots, 0)|^{2}=2 \neq 1$ and $f$ is not defined there. Since

$$
f[1 / 1 \overline{2}, 1 / 1 \overline{2}, 0, \ldots, 0]=2^{-k}
$$

it is enough to find $R f[1 / \sqrt{2}, 1 / \sqrt{2}, 0, \ldots, 0]$.

Under the identification $\mathbf{C} P^{n} \approx \mathbf{C} P^{n *}$ the point $[1 / \sqrt{2}, 1 / \sqrt{2}, 0, \ldots, 0]$ is identified with the hyperplane

$$
H=\left\{\left[z_{0}, \ldots, z_{n}\right] \mid \frac{z_{0}}{\sqrt{2}}+\frac{z_{1}}{\sqrt{2}}=0\right\}=\left\{[z] \mid z_{0}+z_{1}=0\right\} .
$$

In affine coordinates, $H$ is given by the locus $\left\{(t) \mid t_{1}=-1\right\}$. The induced invariant measure on $H$ is

$$
d \mu_{H}=c_{n} \frac{d t_{2} \wedge \cdots \wedge d t_{n} \wedge d \bar{t}_{2} \wedge \cdots \wedge d \bar{t}_{n}}{\left(1+1+\left|t_{2}\right|^{2}+\cdots+\left|t_{n}\right|^{2}\right)^{n}}
$$

which we write as $c_{n} d \tilde{t} \wedge \overline{d \tilde{t}} /\left(2+|\tilde{t}|^{2}\right)^{n}$ (where $c_{n}$ is a constant depending on $n$ only).

As in $\$ 2$ one can derive this form of the measure explicitly from the Fubini-Study metric, or verify an invariance property. Basically, the 2 in $2+|\tilde{t}|^{2}$ is there (rather than 1) because $t_{1}=-1$ on the hyperplane $H$, and the exponent is $n$ rather than $n+1$ because $\operatorname{dim} H=\operatorname{dim} \mathbf{C} P^{n}-1$. (Remember that we assume $n>1$ throughout.) As we shall see below, this form of the measure plays a crucial role in the final result.

What is $f([z])$ as a function of affine coordinates? If $(t)$ are affine coordinates, they correspond to homogeneous coordinates $[1, t]$, or, normalizing,

$$
\left[\frac{1}{\sqrt{1+|t|^{2}}}, \frac{t}{\sqrt{1+|t|^{2}}}\right]
$$

On $H, t_{1}=-1$ so

$$
\left(z_{0} \bar{z}_{1}\right)^{k}=(-1)^{k}\left|z_{0}\right|^{2 k}=\frac{(-1)^{k}}{\left(2+|\tilde{t}|^{2}\right)^{k}}
$$

Thus

$$
\begin{aligned}
R f(H) & =c_{n}(-1)^{k} \int_{\mathbf{C}^{n-1}}\left(2+|\tilde{t}|^{2}\right)^{-(k+n)} d \tilde{t} \wedge d \overline{\tilde{t}} \\
& =c_{n} \operatorname{Vol}\left(s^{2 n-3}\right)(-1)^{k} \int_{0}^{\infty} \frac{r^{2 n-3}}{\left(2+r^{2}\right)^{n+k}} d r
\end{aligned}
$$


To evaluate the last integral we make the trigonometric substitution $r=\sqrt{2} \tan \theta$, $d r=\sqrt{2} \sec ^{2} \theta d \theta$.

$$
\begin{aligned}
R f(H) & =c_{n}(-1)^{k} \int_{0}^{\pi / 2} \frac{(\sqrt{2} \tan \theta)^{2 n-3} \sqrt{2} \sec ^{2} \theta d \theta}{\left(2 \sec ^{2} \theta\right)^{n+k}} \\
& =c_{n}(-2)^{-k} \int_{0}^{\pi / 2}(\cos \theta)^{2(k+1)-1}(\sin \theta)^{2(n-1)-1} d \theta \\
& =c_{n}(-2)^{-k} \frac{1}{(k+1) \cdots(k+n-1)}
\end{aligned}
$$

(integration by parts or beta-integral). Thus

$$
c(k)=c_{n} \frac{(-1)^{k}}{(k+1) \cdots(k+n-1)} .
$$

Many properties of the Radon transform can be read off from this formula. For instance, it follows that $R$ is bounded in the $L^{2}$ norm and so it extends continuously to all of $L^{2}\left(\mathbf{C} P^{n}\right)$. In fact, $R$ can be shown to be a Fourier integral operator of order $-(n-1)$ and hence it extends as a bounded operator between Sobolev spaces. This fact also follows from the general theory of Guillemin (see [6]). We will not pursue these ideas here, but rather aim at an inversion formula for $R$.

As usual, we have the dual Radon transform

$$
R^{t}: C\left(\mathbf{C} P^{n *}\right) \rightarrow C\left(\mathbf{C} P^{n}\right) .
$$

It associates to a function $k(H)$ on hyperplanes, a function $R k(x)$ on points. $R k(x)$ is just the integral of $k(H)$ over the set of all hyperplanes $H$ passing through $X$, with a suitable (invariant) measure. In this setting, identifiying $\mathbf{C} P^{n *}$ with $\mathbf{C} P^{n}$ amounts to identifying $R^{t}$ with $R$.

Thus the iterated transform $R^{t} R$ acts on the space $H_{k, k}$ as the scalar

$$
s(k)=c_{n}\left(\frac{1}{(k+1) \cdots(k+n-1)}\right)^{2} .
$$

Recall that the Laplace-Beltrami operator on $C P^{n}$ has the spectrum $\{4 k(n+k) \mid$ $k=0,1,2, \ldots\}$. If we normalize the Laplacian $L$, we may assume that $H_{k, k}$ is an eigenspace for $L$ with eigenvalue $\lambda_{k}=k(n+k)$. (All this follows easily from the theory of harmonics on $S^{2 n+1}$; see Berger [1].)

We can rearrange the terms $((k+1) \cdots(k+n-1))^{2}$ as

$$
(k+1)(k+n-1)(k+2)(k+n-2) \cdots(k+n-1)(k+1),
$$

and we get

$$
\begin{aligned}
\frac{1}{s(k)} & =c_{n} \prod_{j=1}^{n-1}(k+j)(k+n-j)=c_{n} \prod_{j=1}^{n-1}(k(k+n)+j(n-j)) \\
& =c_{n} \prod_{j=1}^{n-1}\left(\lambda_{k}+j(n-j)\right)=P\left(\lambda_{k}\right)
\end{aligned}
$$


where $P$ is the polynomial $P(A)=c_{n} \prod_{j=1}^{n-1}(A+j(n-j))$. Hence we get the inversion formula

$$
P(L) R^{t} R=I
$$

on any polynomial function, and indeed on all of $L^{2}\left(\mathbf{C} P^{n}\right)$ if we interpret the Laplace-Beltrami operator as a pseudo differential operator between Sobolev spaces (the interpretation on the level of $C^{x}$ functions is clear, and requires little machinery). This verifies the formula in Helgason [8, p. 126] after some renormalizations are accounted for.

To conclude this section, we compute the value of $c_{n}$. If $f(x)$ is the constant function $1, R f$ ought to be the constant function

$$
\operatorname{Vol}\left(\mathbf{C} P^{n-1}\right)=\pi^{n-1} /(n-1) !
$$

(see [1] again). Hence $R^{t} R f$ should be the constant $\left(\pi^{n-1} /(n-1) !\right)^{2}$. Now $P L(1)=$ $((n-1) !)^{2} c_{n}$ so if we let $c_{n}=\pi^{-2 n+2}$ we get $P(L) R^{t} R 1=1$ with

$$
P(L)=\pi^{-2 n+2}(L+1 \cdot(n-1))(L+2 \cdot(n-2)) \cdots(L+(n-1) \cdot 1)
$$

and we have a complete description of the inversion formula.

4. The quaternionic projective space $\mathbf{H} P^{n}$. We will think of a quaternion $q \in \mathbf{H}$ as given by a pair of complex numbers $z$ and $w$ in the following way:

$$
q=z+w j \text {. }
$$

In this way, the quaternion conjugate becomes $\bar{q}=\bar{z}-w j$ and $\|q\|^{2}=q \bar{q}=\bar{q} q=$ $z \bar{z}+w \bar{w}$.

Let $S^{3}=\mathrm{SU}(2)$ be the group of unit quaternions. Then $S^{3}$ acts on affine quaternionic $(n+1)$-space $\mathbf{H}^{n+1}$ by right multiplication:

$$
\begin{gathered}
q=\left(q_{0}, \ldots, z_{n}\right)=\left(\left(z_{0}, w_{0}\right), \ldots,\left(z_{n}, w_{n}\right)\right) \in \mathbf{H}^{n+1}, \\
\lambda \in S^{3} \subset \mathbf{H}, \quad q \lambda=\left(q_{0} \lambda, \ldots, q_{n} \lambda\right) .
\end{gathered}
$$

The quaternionic projective space $\mathbf{H} P^{n}$ can be described as the quotient space $\mathbf{H} P^{n}=\left(\mathbf{H}^{n+1}-0\right) /\left(\mathrm{SU}(2) \times \mathbf{R}^{*}\right)$.

As with $\mathbf{C} P^{n}$, we have homogeneous coordinates $[q]=\left[q_{0}, \ldots, q_{n}\right]$ on $\mathbf{H} P^{n}$, and on the affine open set $U_{i}\left\{[q] \in \mathbf{H} P^{n} \mid q_{i} \neq 0\right\}$ we have affine quaternion coordinates $t_{i}^{j}=q_{j} q_{i}^{-1}(j \neq i)$.

On the overlap $U_{i} \cap U_{k}$ the transition functions are of the type

$$
q_{i} q_{k}^{-1}=\frac{q_{i} \bar{q}_{k}}{\left|q_{k}\right|^{2}}=\frac{\left(z_{i}+w_{i} j\right)\left(\bar{z}_{k}-w_{k} j\right)}{z_{k} \bar{z}_{k}+w_{k} \bar{w}_{k}}
$$

and hence endow $\mathbf{H} P^{n}$ with a real analytic manifold structure, but not a complex analytic structure.

Now $\mathbf{H} P^{n+1}$ is a representation space of the quaternionic unitary group (or symplectic group) $\operatorname{SP}(n+1)$, under left translation:

$$
U \in \operatorname{SP}(n+1), \quad q=\left(q_{0}, \ldots, q_{n}\right) \in \mathbf{H} P^{n+1}, \quad U \circ q=U^{-1}\left(\begin{array}{c}
q_{0} \\
\vdots \\
q_{n}
\end{array}\right) \text {. }
$$


Since the left action of $\operatorname{SP}(n+1)$ commutes with the right action of $\mathrm{SU}(2)$, the $\mathrm{SP}(n+1)$ action pushes forward to an induced action on $\mathbf{H} P^{n}$. Clearly, the action is transitive and we can give $\mathbf{H} P^{n}$ a metric so that $G=\operatorname{SP}(n+1)$ becomes a group of isometries. Thus $\mathbf{H} P^{n}$ may be viewed as the homogeneous space $\operatorname{SP}(n+1) / \operatorname{SP}(n)$ $\times \mathrm{SP}(1)(\mathrm{SU}(2)=\mathrm{SP}(1))$. To study measure theory and representation theory on $\mathbf{H} P^{n}$ we will make use of an analog of the Hopf fibration:

$$
\begin{array}{r}
F \rightarrow \quad \mathbf{C} P^{2 n+1} \\
\downarrow \pi \\
\mathbf{H} P^{n}
\end{array}
$$

where $\pi\left[\alpha_{0}, \beta_{0}, \ldots, \alpha_{n+1}, \beta_{n+1}\right]=\left[\alpha_{0}+\bar{\beta}_{0} j, \ldots, \alpha_{n}+\bar{\beta}_{n} j\right]$.

Multiplication of the homogeneous coordinates on $\mathbf{C} P^{n}$ by the phase factor $e^{i \theta}$ corresponds to right multiplication of the quaternionic homogeneous coordinates by the unit quaternion

$$
\lambda=\left(\begin{array}{cc}
e^{i \theta} & 0 \\
0 & e^{-i \theta}
\end{array}\right) \in \operatorname{SU}(2) .
$$

This gives an imbedding $U(1) \subset \mathrm{SU}(2)$ and shows that the map $\pi$ is well defined. $\pi$ is a fibration with fiber

$$
F=\mathrm{SU}(2) / U(1)=S^{3} / S^{1} .
$$

Let $\rho$ denote the usual Hopf fibration $\rho: S^{4 n+3} \rightarrow \mathbf{C} P^{2 n+1}$. Then $\pi \circ \rho$ gives a fibration $S^{4 n+3} \rightarrow \mathbf{H} P^{n}$ which is the usual quaternionic Hopf fibration. We have the following commutative diagram:

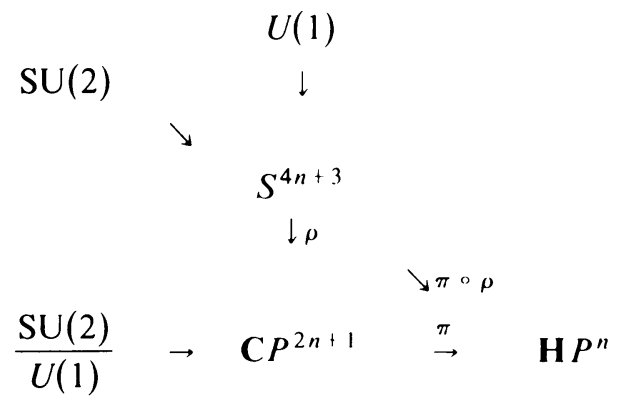

A function $f \in C\left(\mathbf{H} P^{n}\right)$ lifts to a function $\pi^{*} f \in C\left(\mathbf{C} P^{2 n+1}\right)$ which is invariant under the induced right $\mathrm{SU}(2)$ action. This function in turn lifts to a function $\rho^{*} \pi^{*} f \in C\left(S^{4 n+3}\right)$ which is again SU(2) invariant, and in particular, SU(1) invariant. In $\S 1$ we decomposed the space of $L^{2}$ functions on spheres (such as $S^{4 n+3}$ ) invariant under $\mathrm{SU}(1)$ as a representation space for the complex unitary group $U(2 n+2)$. In other words, we decomposed the representation of $U(2 n+2)$ induced from the trivial representation of $U(n) \times U(1)$. Here we need to decompose the $U(2 n+2)$ representation induced from the trivial representation of $U(2 n) \times \operatorname{SU}(2)$. Our discussion closely parallels that of $\S 1$.

Let $[q]=\left[q_{0}, \ldots, q_{n}\right]=\left[z_{0}, w_{0}, \ldots, z_{n}, w_{n}\right] \in \mathbf{H} P^{n}$. We first find polynomials in $z_{i}, w_{j}, \bar{z}_{i}, \bar{w}_{j}$ which are $\mathrm{SU}(2)$ invariant (throughout the discussion, we restrict to $\left(z_{0}, \ldots, w_{n}\right) \in S^{4 n+3}$ of course). There are two ways to obtain such polynomials, 
inner products and determinants:

$$
\begin{aligned}
q_{0} \bar{q}_{1} & =\left(z_{0}+w_{0} j\right)\left(\bar{z}_{1}-w_{1} j\right)=\left(z_{0} \bar{z}_{1}+w_{0} \bar{w}_{1}\right)-\left(z_{0} w_{1}-z_{1} w_{0}\right) j \\
& =\left\langle\left(z_{0}, w_{0}\right),\left(z_{1}, w_{1}\right)\right\rangle-\operatorname{det}\left(\begin{array}{cc}
z_{0} & z_{1} \\
w_{0} & w_{1}
\end{array}\right) j .
\end{aligned}
$$

Since the quaternion function $q_{0} \bar{q}_{1}$ is invariant under right multiplication by $\operatorname{SU}(2)$ (of the argument $\left(q_{0}, \ldots, q_{n}\right)$ ), so are the complex polynomials $z_{0} \bar{z}_{1}+w_{0} \bar{w}_{1}$ and $z_{0} w_{1}-z_{1} w_{0}$. (The second polynomial lifts to $\mathbf{C} P^{2 n+1}$ to become $z_{0} \bar{w}_{1}-z_{1} \bar{w}_{0}$, while the first becomes $z_{0} \bar{z}_{1}+w_{1} \bar{w}_{0}$.) It can be proved by means of invariant theory (see Weyl [12]) that these generate the ring of invariants for $\mathrm{SU}(2)$ on polynomials in $z_{0}, \ldots, w_{n}$. However, we will not need the full strength of this result and so we will not prove it. In fact, we will discard the invariants generated by $z_{i} w_{j}-z_{j} w_{i}$ (which do not form a selfadjoint algebra) and concentrate on the $z_{i} \bar{z}_{j}+w_{i} \bar{w}_{j}$.

Let $A_{m}(n+1)$ be the vector spaces of all homogeneous polynomials over $\mathbf{C}$ of degree $m$ in the invariants $\left\{z_{l} \bar{z}_{1}+w_{1} \bar{w}_{l} \mid 0 \leqslant i, j \leqslant n\right\}$ viewed as functions on $\mathbf{H} P^{n}$. Put $A=\bigoplus_{m} A_{m}$ (inductive sum).

Then $A$ is a selfadjoint algebra of continuous functions on $\mathbf{H} P^{n}$ which, as in $\S 1$, vanishes nowhere and separates points. Hence it is dense in $L^{2}\left(\mathbf{H} P^{n}\right)$. We now consider the quaternionic Laplacian

$$
\Delta \sum_{1}^{n}\left(\frac{\partial^{2}}{\partial z_{1} \partial \bar{z}_{1}}+\frac{\partial^{2}}{\partial w_{l} \partial \bar{w}_{l}}\right)
$$

and put $H_{m}(n+1)=A_{m}(n+1) \cap \operatorname{Ker} \Delta$, i.e., harmonic polynomials of degree $m$ in the invariants $z_{1} \bar{z}_{1}+w_{1} \bar{w}_{1}$. Each $H_{m}(n+1)$ is (left) $\operatorname{SP}(n+1)$-invariant. We can prove that each space $H_{m}$ is irreducible under $G=\operatorname{SP}(n+1)$, and that the representations are pairwise inequivalent. The argument is similar to that of $\S 1$; details are left to the reader. Thus we have

$$
L^{2}\left(\mathbf{H} P^{n}\right)=\bigoplus_{m=0}^{\infty} H_{m}(n+1) \quad\left(\text { as an orthogonal } L^{2} \text { decomposition }\right)
$$

Also, if $S$ is the hyperplane $\left\{[q] \in \mathbf{H} P^{n} \mid q_{0}+q_{1}=0\right\}$, then the induced invariant measure on it is just

$$
c_{n} \frac{d \tilde{z}_{2} \wedge d \overline{\tilde{x}}_{2} \wedge d \tilde{w}_{2} \wedge d \overline{\tilde{w}}_{2} \wedge \cdots \wedge d \overline{\tilde{w}}_{n}}{\left(2+\left|\tilde{z}_{2}\right|^{2}+\cdots+\left|\tilde{w}_{n}\right|^{2}\right)^{2 n}}=\frac{d \tilde{q}_{1} \wedge d \overline{\tilde{q}}_{1} \wedge \cdots \wedge d \overline{\tilde{q}}_{n}}{\left(2+|\tilde{q}|^{2}\right)^{2 n}}
$$

in complete analogy with the complex case.

5. The quaternionic Radon transform. The discussion of the Radon transform on $\mathbf{C} P^{n}$ in $\S 3$ goes through almost verbatim so we go straight to the calculations.

We select a function $f(z, w) \in H_{k}(n+1)$ and compare $R f$ with $f$. Let $f(z, w)=$ $\left(z_{0} \bar{z}_{1}+w_{0} \bar{w}_{1}\right)^{k} \in H_{k}$. Again, this is a highest weight vector and is easy to integrate. 
Now $f([1 / \sqrt{2}, 1 / \sqrt{2}, 0, \ldots, 0])=2^{-k}$. We find $R f[1 / \sqrt{2}, 1 / \sqrt{2}, 0, \ldots, 0]$. This amounts to evaluating the integral

$$
\begin{aligned}
c_{n} \int_{\mathbf{C}^{2(n-1)}} \frac{(-1)^{k}}{\left(2+|t|^{2}\right)^{k+2 n} d t \wedge d \bar{t}} & =c_{n} \operatorname{Vol}\left(S^{4 n-5}\right)(-1)^{k} \int \frac{r^{4 n-5}}{\left(2+r^{2}\right)^{2 n+k}} d r \\
& =c_{n}(-2)^{-k} \int_{0}^{\pi / 2}(\cos \theta)^{2(k+2)-1}(\sin \theta)^{2(2 n-2)-1} d \theta \\
& =c_{n}(-2)^{-k} \frac{1}{(k+2)(k+3) \cdots(k+2 n-1)},
\end{aligned}
$$

so as before, the Radon transform acts on $H_{k}$ as the scalar

$$
c(k)=c_{n} \frac{(-1)^{k}}{(k+2) \cdots(k+2 n-1)}
$$

and $R^{t} R$ acts as

$$
s(k)=c^{2}(k)=c_{n} \frac{1}{((k+2) \cdots(k+2 n-1))^{2}} .
$$

We write

$$
\begin{aligned}
((k+ & 2) \cdots(k+2 n-1))^{2} \\
& =[(k+2)(k+2 n-1)][(k+3)(k+2 n-2)] \cdots[(k+2 n-1)(k+2)] \\
& =\sum_{j=2}^{2 n-1}(k+j)(k+2 n+1-j) \\
& =\sum_{j=2}^{2 n-1}[k(k+2 n+1)-j k+j(k+2 n+1-j)] \\
& =\sum_{j=2}^{2 n-1}\left[\lambda_{k}+j(2 n+1-j)\right]
\end{aligned}
$$

where $\lambda_{k}=k(k+2 n+1)$ is the eigenvalue of the $\mathbf{H} P^{n}$ Laplace-Beltrami operator $L$ on $H_{k}$ (after renormalization). As before, if we define the polynomial

$$
P(L)=(L+2(2 n-1))(L+3(2 n-2)) \cdots(L+(2 n-1) \cdot 2) \cdot c_{n}
$$

we have the inversion formula $P(L) R^{t} R=I$ which again verifies Helgason's formula after renormalization.

To determine $c_{n}$ we observe that $P(L)\left(1_{\mathbf{H} P^{n}}\right)=((2 n-1) !)^{2}$ and

$$
\operatorname{Vol}\left(\mathbf{H} P^{n}\right)=\frac{\pi^{2 n}}{(2 n+1) !}
$$

(Berger [1, p. 112]). Now $R\left(1_{\mathbf{H} P^{n}}\right)$ should be the constant function $\operatorname{Vol}\left(\mathbf{H} P^{n-1}\right)$ so we have

$$
\frac{c_{n}((2 n-1) !)^{2}\left(\pi^{2 n-2}\right)^{2}}{((2 n-1) !)^{2}}=1
$$

so $c_{n}=\pi^{4-4 n}$ and we have a complete description of $P$. 
6. The Cayley projective plane. There remains one example of a projective space (or compact two-point homogeneous space) for us to examine. It is a homogeneous space of the group $F_{4}$, namely $F_{4} / \operatorname{Spin}(9)$, and its construction is based on the Cayley numbers (or octonians). Since explicit constructions of this manifold as a projective space are hard to find in the literature, we will discuss its structure in detail. We refer to the appendix of [13] for general information about octonians; [10] may also be helpful.

We view an octonian $\theta$ as a pair of quaternions $(a, b)$. With this notation the Cayley multiplication and conjugation is defined as follows:

$$
(a, b)(r, s)=(a r-\bar{s} b, s a+b \bar{r}), \quad(\overline{a, b})=(\bar{a},-b) .
$$

The octonians $\mathbf{O}$ form a nonassociative, noncommutative 8-dimensional algebra over $\mathbf{R}$. To define a projective plane $\mathbf{O} P^{2}$ over $\mathbf{O}$, we can try the usual method of homogeneous coordinates. Thus we would like $\mathbf{O} P^{2}$ to be the set of all homogeneous coordinates $\left[\theta_{0}, \theta_{1}, \theta_{2}\right]$ (with $\left(\theta_{0}, \theta_{1}, \theta_{2}\right) \in \mathbf{O}^{3}-0$, or $\left.S^{23}\right)$ and the identification

$$
\left[\theta_{0}, \theta_{1}, \theta_{2}\right]=\left[\theta_{0} \lambda, \theta_{1} \lambda, \theta_{2} \lambda\right] \quad(\lambda \in \mathbf{O}-0) .
$$

However, because of the lack of associativity, this does not induce an equivalence relation (the transitive law fails). We could try to get around this by taking the equivalence relation generated by the relation defined above. But this identifies too many points and we would end up having certain $\left[\theta_{0}, \theta_{1}, \theta_{2}\right]=\left[\varphi_{0}, \varphi_{1}, \varphi_{2}\right]$ although $\left(\theta_{0}, \theta_{1}, \theta_{2}\right) \neq\left(\varphi_{0} \lambda, \varphi_{1} \lambda, \varphi_{2} \lambda\right)$ for any $\lambda \in \mathbf{O}$.

Another approach to try is that of affine coordinates: we can take three copies of the affine space $\mathbf{O}^{2}$ and glue them together. Let

$$
U_{0}=\left\{\left[1, \theta_{1}, \theta_{2}\right] \mid \theta_{i} \in \mathbf{O}\right\}, \quad U_{1}=\left\{\left[\theta_{0}, 1, \theta_{2}\right]\right\} . \quad U_{2}=\left\{\left[\theta_{0}, \theta_{1}, 1\right]\right\} .
$$

We identify some points in $U_{0}$ and $U_{1}$ as follows:

$$
\left[1, \theta_{1}, \theta_{2}\right]=\left[\theta_{1}^{-1}, 1, \theta_{2} \theta_{1}^{-1}\right] \quad\left(\theta_{1} \neq 0\right),
$$

and

$$
\left[\theta_{0}, 1, \theta_{2}\right]=\left[1, \theta_{0}^{-1}, \theta_{2} \theta_{0}^{-1}\right] \quad\left(\theta_{0} \neq 0\right) .
$$

Similar identifications are made between $U_{0}$ and $U_{2}$ and $U_{1}$ and $U_{2}$. We need to check that the implied relation is symmetric and transitive, i.e., that first, $\left[1, \theta_{1}\right.$, $\left.\theta_{2}\right]=\left[\theta_{1}^{-1}, 1, \theta_{2} \theta_{1}^{-1}\right]=\left[1, \theta_{1},\left(\theta_{2} \theta_{1}^{-1}\right) \theta_{1}\right]$ is consistent (i.e., $\left.\theta_{2}=\left(\theta_{2} \theta_{1}^{-1}\right) \theta_{1}\right)$, and, second, that on triple overlaps going from $U_{0}$ to $U_{2}$ directly is the same as going through $U_{1}$ :

$$
\begin{aligned}
{\left[1, \theta_{1}, \theta_{2}\right] } & =\left[\theta_{1}^{-1}, 1, \theta_{2} \theta_{1}^{-1}\right]=\left[\theta_{1}^{-1}\left(\theta_{1} \theta_{2}^{-1}\right), \theta_{1} \theta_{2}^{-1}, 1\right] \\
& =\left[\theta_{2}^{-1}, \theta_{1} \theta_{2}^{-1}, 1\right] \quad\left(\theta_{i} \neq 0\right)
\end{aligned}
$$

(i.e., $\left.\theta_{2}^{-1}=\left(\theta_{1}\right)^{-1}\left(\theta_{1} \theta_{2}^{-1}\right)\right)$.

All of these requirements (and many others) can be verified from the following fundamental fact (see [13]).

THEOREM. The Cayley numbers form an alternative algebra. In other words, any two octonians generate an associative subalgebra. 
Knowing this we can redefine $\mathbf{O} P^{2}$ more elegantly by taking the set of all homogeneous coordinates $\left[\theta_{0}, \theta_{1}, \theta_{2}\right]$ such that $\left\{\theta_{0}, \theta_{1}, \theta_{2}\right\}$ generate an associative subalgebra $\mathbb{Q}\left(\theta_{0}, \theta_{1}, \theta_{2}\right) \subset \mathbf{O}$ and identifying $\left[\theta_{0}, \theta_{1}, \theta_{2}\right]$ with $\left[\theta_{0} \lambda, \theta_{1} \lambda, \theta_{2} \lambda\right]$ whenever $\lambda \in \mathbb{Q}\left(\theta_{0}, \theta_{1}, \theta_{2}\right) \backslash 0$. The theorem also shows why $\mathbf{O} P^{2}$ is the highestdimensional projective space we can define over $\mathbf{O}$. For example, to define $\mathbf{O} P^{3}$ we would have to verify a consistency condition on quadruple overlaps. This essentially requires an associativity condition among three arbitrary octonians, which fails of course. (The reader may find it instructive to write out the precise relations needed.) Finally, we note that the first projective space $O P^{1}$ can be defined just like $O P^{2}$ : take homogeneous coordinates $\left[\theta_{0}, \theta_{1}\right]$, etc. We have the classifical identifications $\mathbf{R} P^{1} \approx S^{1}, \mathbf{C} P^{1} \approx S^{2}, \mathbf{H} P^{1} \approx S^{4}$ and fortunately the list can be completed with $\mathbf{O} P^{1} \approx S^{8}$ (see Whitehead [13]).

For the projective spaces $\mathbf{R} P^{n}, \mathbf{C} P^{n}$, and $\mathbf{H} P^{n}$ we have the Hopf fibrations

$$
S^{n} \rightarrow \mathbf{R} P^{n}, \quad S^{2 n+1} \rightarrow \mathbf{C} P^{n}, \quad S^{4 n+3} \rightarrow \mathbf{H} P^{n},
$$

and so we expect a fibration $S^{8 n+7} \rightarrow \mathbf{O} P^{n}(n=1,2)$. The first of these, $S^{15} \rightarrow \mathbf{O} P^{1}$ $=S^{8}$ can be realized as follows: we think of $S^{15}$ as the unit sphere in $\mathbf{O}^{2}$, and then we have the map

$$
\left(\theta_{0}, \theta_{1}\right) \rightarrow\left[\theta_{0}, \theta_{1}\right] \quad\left(\theta_{0} \bar{\theta}_{0}+\theta_{1} \bar{\theta}_{1}=1\right) .
$$

The second fibration, $S^{23} \rightarrow \mathbf{O} P^{2}$, cannot be realized, unfortunately. Our method does not extend because $\left(\theta_{0}, \theta_{1}, \theta_{2}\right)$ may be a nonassociative triplet of octonians, so that the homogeneous coordinates $\left[\theta_{0}, \theta_{1}, \theta_{2}\right]$ are forbidden. Thus we cannot proceed as in the previous cases pulling everything back to a sphere. Still, as we shall see, all the important invariants behave as if they were derived from an appropriate sphere.

The first thing we need to do is to realize $\mathbf{O} P^{2}$ as a homogeneous space. Clearly, octonian-linear motions will not work here (left and right multipliciations do not commute). Instead, we embed $\mathbf{O} P^{2}$ in $\mathbf{R}^{27}$ as follows:

$$
\left[\theta_{0}, \theta_{1}, \theta_{2}\right] \rightarrow\left(\xi_{1}, \xi_{2}, \xi_{3}, x_{1}, x_{2}, x_{3}\right) \in \mathbf{R}^{3} \times \mathbf{O}^{3} \approx \mathbf{R}^{27},
$$

where $\xi_{i}=\theta_{i} \bar{\theta}_{i}(i=0,1,2), x_{1}=\theta_{1}, \bar{\theta}_{2}, x_{2}=\theta_{2} \bar{\theta}_{0}$, and $x_{3}=\theta_{0} \bar{\theta}_{1}$.

Here and henceforth we normalize all our homogeneous coordinates $\left[\theta_{0}, \theta_{1}, \theta_{2}\right]$ so that $\theta_{0} \bar{\theta}_{0}+\theta_{1} \bar{\theta}_{1}+\theta_{2} \bar{\theta}_{2}=1$, and the functions $\xi_{i}, x_{j}$ are well defined. It is easy to verify that this gives us an embedding; see [10, p. 416]. With the vector $\left(\xi_{i}, x_{j}\right)$ we associate the $3 \times 3$ matrix of octonians.

$$
\left(\begin{array}{lll}
\xi_{1} & x_{3} & \bar{x}_{2} \\
\bar{x}_{3} & \xi_{2} & x_{1} \\
x_{2} & \bar{x}_{1} & \xi_{3}
\end{array}\right)
$$

in which the diagonal entries are real. Such matrices form an (exceptional) Jordan algebra $\mathfrak{\Im}$ under the multiplication $X^{*} Y=\frac{1}{2}(X Y+Y X)$ (where $X Y$ is the octonianmatrix product). 
The image matrices of our embedding of $\mathbf{O} P^{2}$ satisfy the following relations:

$$
\begin{aligned}
& x_{2} x_{3}=\xi_{1} \bar{x}_{1}, \quad\left\|x_{1}\right\|^{2}=\xi_{2} \xi_{3}, \\
& x_{3} x_{1}=\xi_{2} \bar{x}_{2}, \quad\left\|x_{2}\right\|^{2}=\xi_{3} \xi_{1}, \quad \xi_{1}+\xi_{2}+\xi_{3}=1 \text {. } \\
& x_{1} x_{2}=\xi_{3} \bar{x}_{3}, \quad\left\|x_{3}\right\|^{2}=\xi_{1} \xi_{2},
\end{aligned}
$$

In fact, these relations characterize the range of our embedding. Thus we realize $\mathbf{O} P^{2}$ as the set of primitive idempotents in $i$. The automorphism group of the Jordan algebra $\mathfrak{J}$ is the exceptional group $F_{4}$. It acts transitively on the primitive idempotents $\mathbf{O} P^{2}$ and so gives the homogeneous structure. Evidently, the group of isometries acts nonlinearly on the homogeneous coordinates, unlike in the associative cases. But there is a related linear action, which is what we really need.

We can think of an octonian $\theta$ as a quadruplet of complex numbers $(z, w, u, v)$. Thus the six octonian-valued functions $x_{1}, x_{2}, x_{3}, \bar{x}_{1}, \bar{x}_{2}, \bar{x}_{3}$ give rise to 24 complex valued functions on $\mathbf{O} P^{2}$. These, along with the $\xi_{i}$ 's form a 27-dimensional (complex) vector space $V$ of functions. The action of $F_{4}$ on $\mathbf{O} P^{2}$ by isometries induces a linear action on this vector space. We can think of $V$ as consisting of polynomial functions in the sphere of $\left(\mathbf{C}^{4}\right)^{3} \approx\left(\mathbf{R}^{8}\right)^{3} \approx \mathbf{O}^{3}$. As before, we can define a Laplacian, the Cayley-Laplacian

$$
\Delta_{\mathbf{O}}=\frac{\partial^{2}}{\partial z \partial \bar{z}}+\frac{\partial^{2}}{\partial w \partial \bar{w}}+\frac{\partial^{2}}{\partial u \partial \bar{u}}+\frac{\partial^{2}}{\partial v \partial \bar{v}}
$$

and consider harmonic polynomials in the generators of $V$. Thus we get an algebra ( $c^{\prime}$ with the Stone-Weierstrass property. Now $F_{4}$ acts on $\mathbf{O} P^{2}$ and the isotropy group of a point $\mathfrak{v}, \operatorname{Spin}(9)$, acts transitively on $\mathbf{O} P^{1}$. Using this fact we can deduce the existence and uniqueness of spherical functions in homogeneous parts of $C^{\prime}$, and thereby obtain an invariant irreducible decomposition of $L^{2}\left(\mathbf{O} P^{2}\right)$. Indeed, the analysis from this point on is analogous to the previous cases so we omit the details.

What is the spectrum of $\mathbf{O} P^{2}$ ? The other projective spaces have (renormalized) spectra as follows:

\begin{tabular}{c|c}
$X$ & $\operatorname{Spec}(X)$ \\
\hline $\mathbf{R} P^{n}$ & $k(k+n-1)$ \\
$\mathbf{C} P^{n}$ & $k(k+n)$ \\
$\mathbf{H} P^{n}$ & $k(k+2 n+1)$
\end{tabular}

so we expect $\operatorname{Spec}(X)$ to be $k(k+4 n+3)$, or $k(k+11)$ for $\mathbf{O} P^{2}$. This is indeed the case, since our polynomials can be viewed as special functions on $S^{23}$ and the action of $F_{4}$ on the level of functions is compatible with this view.

Finally, the invariant measure on the 'hyperplane' $H=\left\{\left[\theta_{0}, \theta_{1}, \theta_{2}\right] \mid \theta_{0}+\theta_{1}=0\right\}$ in affine coordinates is just what we expect it to be (the explicit action of $F_{4}$ described above can be used to verify its invariance):

$$
d \mu_{H}=\frac{c d \tilde{\theta}_{2} \wedge d \tilde{\overline{\theta_{2}}}}{\left(2+|\tilde{\theta}|^{2}\right)^{8}} .
$$


The scalar by which the Radon transform acts on the $k$ th eigenspace of the Laplace-Beltrami operator $L$ is

$$
c(k)=c \frac{(-1)^{k}}{(k+4)(k+5)(k+6)(k+7)}
$$

and this implies the inversion formula

$$
\pi^{8}(L+28)^{2}(L+30)^{2} R^{t} R=I
$$

which is again a renormalized version of Helgason's formula (we chose the normalization here to make the formula look similar to the previous one).

\section{REFERENCES}

1. M. Berger et al., Le spectre d'une variété Riemannienne. Lecture Notes in Math.. vol. 194. Springer-Verlag, Berlin and New York, 1971.

2. J. Dieudonné, Special functions and linear representations of Lie groups. CBMS Regional Conf. Ser. in Math., no. 42, Amer. Math. Soc., Providence, R.I., 1980.

3. I. M. Gelfand, M. I. Graev and Z. Ya. Shapiro, Differential forms and integral geometry, Functional Anal. Appl. 3 (1969), 24-40.

4. I. M. Giclfand, M. I. Graev and N. Ya. Vilenkin. Generalized functions, vol. 5. Academic Press, New York, 1966.

5. V. Guillemin, Radon transform on Zoll surfaces, Adv. in Math. 22 (1976), 85-119.

6. V. (iuillemin and D. Schaeffer, Fourier integral operators from the Radon transform point of vien: Proc. Sympos. Pure Math., vol. 27. Amer. Math. Soc., Providence, R.I., 1975. pp. 297-300.

7. S. Helgason. The Radon transform on Euclidean spaces, compact two-point homogeneous spaces and (irassmann manifolds, Acta Math. 113 (1965).

8. _. The Radon transform, Progress in Math., Birkhäuser, Basel, 1980.

9. S. Kobayashi and K. Nomizu, Foundations of differential geometry, vols. 1,2, Wiley (Interscience), New York, 1969.

10. I. R. Porteous, Topological geometry, Cambridge Univ. Press, New York, 1981

11. R. T. Smith, The spherical representation of groups transitive on $S^{n}$, Indiana Univ. Math. J. 24 (1974), 307-325.

12. H. Weyl, The classical groups, their invariants and representations, Princeton Univ. Press, Princeton, N.J., 1939

13. (j. W. Whitehead, Elements of homotopy theory, Springer-Verlag, Berlin and New York, 1978.

14. R. S. Strichartz, L." estimates for Radon transforms..., Duke Math. J. 48 (1981), 699-727.

Department of Mathematics, Harvard University, Cambridge, Massachusetts 02138 